\title{
On prime-valent symmetric Cayley graphs of finite simple groups
}

\author{
Jing Jian $\mathrm{Li}^{*}$ \\ College of Mathematics and Information Science \\ Guangxi University \\ Nanning, P. R. China \\ lijjhx@163.com \\ Jicheng $\mathrm{Ma}^{\dagger}$ \\ Chongqing Key Lab. of Group \& Graph Theories and Applications \\ Chongqing University of Arts and Sciences \\ Chongqing, P. R. China \\ ma_jicheng@hotmail.com
}

Submitted: Feb 18, 2020; Accepted: Jan 30, 2021; Published: Feb 26, 2021

(C) The authors. Released under the CC BY-ND license (International 4.0).

\begin{abstract}
We give a characterization of the automorphism groups of connected primevalent symmetric Cayley graphs on finite (non-abelian) simple groups.
\end{abstract}

Mathematics Subject Classifications: 05C25, 05E18

\section{Introduction}

Throughout this paper, all graphs are assumed to be finite, simple and undirected. For a graph $\Gamma$, we denote by $V(\Gamma), E(\Gamma), A(\Gamma)$ and $\operatorname{Aut}(\Gamma)$ its vertex set, edge set, arc set and (full) automorphism group, respectively. A graph $\Gamma$ is said to be $X$-arc-transitive or $X$-symmetric if $X \leqslant \operatorname{Aut}(\Gamma)$ acts transitively on $A(\Gamma)$. Especially, when $X=\operatorname{Aut}(\Gamma)$, an $X$-arc-transitive (or $X$-symmetric) graph is simply called an arc-transitive (or symmetric) graph.

Let $G$ be a group and an inverse-closed subset $S$ of $G \backslash\{1\}$. A Cayley graph Cay $(G, S)$ of $G$ with connection set $S$ is the graph with vertex set $G$ and edge set $\{\{g, s g\} \mid g \in$ $G, s \in S\}$. Clearly, $\operatorname{Cay}(G, S)$ has valency $|S|$, and it is connected if and only if $\langle S\rangle=G$.

\footnotetext{
* Supported by NNSF of China (11861012).

†The corresponding author.
} 
Moreover, each $g \in G$ induces an automorphism of $\operatorname{Cay}(G, S)$ by right multiplication on vertices, and so $G$ can be regarded as a regular subgroup of $\operatorname{Aut}(\operatorname{Cay}(G, S))$. In this way, if $G$ is normal in $\operatorname{Aut}(\operatorname{Cay}(G, S))$, then $\operatorname{Cay}(G, S)$ is called a normal Cayley graph, otherwise it is called a non-normal Cayley graph.

Define

$$
\operatorname{Aut}(G, S)=\left\{\sigma \in \operatorname{Aut}(G) \mid S^{\sigma}=S\right\}
$$

Then it is easy to see that $G: \operatorname{Aut}(G, S) \leqslant \operatorname{Aut}(\operatorname{Cay}(G, S))$. In fact, $G: \operatorname{Aut}(G, S)$ is the normalizer of $G$ in $\operatorname{Aut}(\operatorname{Cay}(G, S)$ ) (see for example [10, 24]). Thus normal Cayley graphs are precisely those $\operatorname{Cay}(G, S)$ with $\operatorname{Aut}(\operatorname{Cay}(G, S))=G$ :Aut $(G, S)$. Hence, the normality is crucial in determining the full automorphism group of a Cayley graph.

The normality of Cayley graphs of finite non-abelian simple groups has received considerable attention $[5,6,7,9,17,25,26]$. In this paper, we focus on symmetric Cayley graphs of prime valency on non-abelian simple groups. This work is motivated by the study of the case when the graph is cubic or pentavalent started by $\mathrm{Li}$ [17] and Fang et al. [7], respectively. In 1996, Li [17] listed all possible finite non-abelian simple groups on which a connected cubic symmetric Cayley graph might be non-normal. Li's list was later made explicit by $\mathrm{Xu}$, Fang, Wang and $\mathrm{Xu}$ [25], who showed that there exists a connected cubic symmetric non-normal Cayley graph on a finite non-abelian simple group $G$ if and only if $G=\mathrm{A}_{47}$. For connected pentavalent symmetric non-normal Cayley graphs on finite non-abelian groups, Fang, Ma and Wang first gave a characterization in 2011 [7]. Then recently Du, Feng and Zhou [5] obtained a list of all possible such non-abelian simple groups. To extend the above results to symmetric Cayley graphs of prime valency $p$ on finite simple groups, we deal with the case when prime $p \geqslant 7$. Note that, if the regular simple group is abelian, say $\mathbf{Z}_{q}$ with prime $q$. Then as each symmetric Cayley graph of prime valency is of even order, thus $q=2$, which implies that $p=1$, a contradiction. Hence, one can only consider the non-abelian simple groups. Our main theorem in the following is a characterization of those possible non-normal ones. For undefined terms, see Section 2.

Theorem 1. Let $G$ be a finite non-abelian simple group, let $\Gamma=\operatorname{Cay}(G, S)$ be a connected $p$-valent symmetric Cayley graph on $G$ with prime $p \geqslant 7$. Then, for $\alpha \in V(\Gamma)$, we have either $\operatorname{Aut}(\Gamma)=G \rtimes \operatorname{Aut}(G, S)$ or one of the following holds:

(a) $\operatorname{Aut}(\Gamma)$ is an almost simple group with socle $L>G$, and $L$ is either a classical simple group or $\left(L, G, L_{\alpha}\right)$ lies in Table 1 ; or

(b) $\operatorname{Aut}(\Gamma)$ has an intransitive non-trivial normal subgroup $K$ such that $\operatorname{Aut}(\Gamma) / K$ is almost simple with socle $\bar{L} \geqslant G K / K \cong G$. Moreover, we have $\bar{L}$ is either a classical simple group or $\left(\bar{L}, G, \bar{L}_{\bar{\alpha}}\right)$ lies in Table 2 , where $\bar{\alpha}$ is a vertex of the quotient graph $\Gamma_{K}$; or $\left(\operatorname{Aut}(\Gamma), G, \operatorname{Aut}(\Gamma)_{\alpha}\right)$ lies in Table 3 .

Remark 2. For line 1 of Table 1, we shall see in Example 3 that there exists a connected non-normal symmetric Cayley graph on $\mathrm{M}_{22}$ of valency 23. For line 2 in Table 1, it is shown in [7, Theorem 1.3] that there exists a connected non-normal symmetric Cayley graph on $\mathrm{A}_{p-1}$ of valency $p$ for each prime $p \geqslant 7$. 
Table 1:

\begin{tabular}{l|l|l|l|l}
\hline & $L$ & $G$ & $L_{\alpha}$ & remark \\
\hline 1 & $\mathrm{M}_{23}$ & $\mathrm{M}_{22}$ & $\mathrm{C}_{23}$ & $p=23$ \\
\hline 2 & $\mathrm{~A}_{n}$ & $\mathrm{~A}_{n-1}$ & {$[n]$} & $p$ divides $n$ \\
\hline 3 & $\mathrm{~A}_{p+1}$ & {$[p+1]$} & $\mathrm{A}_{p}$ & $\Gamma=\mathrm{K}_{p+1}$ \\
\hline 4 & $\mathrm{~A}_{p+3}$ & $\operatorname{PSL}(2, q)$ & $\mathrm{S}_{p}$ & $p=q-2$ for $q$ odd \\
\hline
\end{tabular}

Table 2:

\begin{tabular}{l|l|l|l|l|l}
\hline & $\bar{L}$ & $G$ & $\bar{L}_{\bar{\alpha}}$ & $K$ & remark \\
\hline 1 & $\mathrm{~A}_{n}$ & $\mathrm{~A}_{n-1}$ & $\begin{array}{l}\text { has a subgroup } \\
\text { of index } n\end{array}$ & & $p$ divides $n$ \\
\hline 2 & $\mathrm{~A}_{p}$ & $\mathrm{~A}_{p-2}$ & $\operatorname{PGL}(d, q) \cdot\langle\sigma\rangle$ & & $\begin{array}{l}p=\left(q^{d}-1\right) /(q-1), \\
\sigma \text { divides } f\end{array}$ \\
\hline 3 & $\mathrm{~A}_{p}$ & $\mathrm{~A}_{p-3}$ & $\operatorname{PGL}(2, q) \cdot\langle\sigma\rangle$ & & $p=q+1, \sigma$ divides $f$ \\
\hline 4 & $\mathrm{~A}_{p}$ & $\mathrm{~A}_{p-3}$ & $\mathrm{AGL}(d, 2)$ & & $p=2^{d}-1$ for $d$ odd \\
\hline 5 & $\mathrm{~A}_{p+1}$ & $\begin{array}{l}\text { has a subgroup } \\
\text { of index } p+1\end{array}$ & $\mathrm{~A}_{p}$ & & $\Gamma_{K}=\mathrm{K}_{p+1}$ \\
\hline 6 & $\mathrm{~A}_{p+3}$ & $\operatorname{PSL}(2, p+2)$ & $\mathrm{S}_{p}$ & & \\
\hline 7 & $\mathrm{~A}_{p+k}$ & $\operatorname{PSL}(d, q)$ & $\mathrm{A}_{p}$ or $\mathrm{S}_{p}$ & & $\frac{q^{d}-1}{q-1}=p+k, k=2$ or 3 \\
\hline 8 & $\mathrm{~A}_{23}$ & $\mathrm{M}_{23}$ & $\mathrm{~A}_{19}$ & {$[48]$} & \\
& & & $\mathrm{S}_{19}$ & {$[96]$} & \\
\hline
\end{tabular}

Table 3:

\begin{tabular}{l|l|l|l|l}
\hline & $\operatorname{Aut}(\Gamma)$ & $G$ & $\operatorname{Aut}(\Gamma)_{\alpha}$ & $\Gamma_{K}$ \\
\hline 1 & $\operatorname{PSL}(2,11) \times \mathrm{M}_{12}$ & $\mathrm{M}_{11}$ & $\mathrm{M}_{11}$ & $\mathrm{~K}_{12}$ \\
\hline 2 & $\left(\mathrm{C}_{11}: \mathrm{C}_{5}\right) \times \mathrm{M}_{12}$ & $\operatorname{PSL}(2,11)$ & $\mathrm{M}_{11}$ & $\mathrm{~K}_{12}$ \\
\hline 3 & $\mathrm{C}_{5} \times \mathrm{M}_{12}$ & $\mathrm{~A}_{5}$ & $\mathrm{M}_{11}$ & $\mathrm{~K}_{12}$ \\
\hline 4 & $\mathrm{C}_{11} \times \mathrm{M}_{23}$ & $\mathrm{M}_{22}$ & $\mathrm{C}_{23}: \mathrm{C}_{11}$ & \\
\hline 5 & $\left(\mathrm{C}_{23}: \mathrm{C}_{11}\right) \times \mathrm{M}_{24}$ & $\operatorname{PSL}(2,23)$ & $\mathrm{M}_{23}$ & $\mathrm{~K}_{24}$ \\
\hline 6 & $\left(\mathrm{C}_{7}: \mathrm{C}_{3}\right) \times \operatorname{AGL}(3,2)$ & $\operatorname{PSL}(2,7)$ & $\mathrm{SL}(3,2)$ & $\mathrm{K}_{8}$ \\
\hline
\end{tabular}




\section{Preliminaries}

Let $G$ be a finite group, denote by $\pi(G)$ the set of prime divisors of $|G|$, by $M(G)$ the Schur multiplier of $G$, and by $\operatorname{Soc}(G)$ the socle (that is, the product of all the minimal normal subgroups) of $G$. We say $G$ is almost simple if $\operatorname{Soc}(G)$ is non-abelian simple. Let $n$ be a positive integer, denote by $[n]$ an (unspecified) group of order $n$, by $\mathrm{F}_{n}$ a Frobenius group of order $n$, by $\mathrm{D}_{2 n}$ the dihedral group of order $2 n$, and by $\mathrm{K}_{n}$ the complete graph of order $n$. For a prime number $r$, let $n_{r}$ be the largest power of $r$ dividing $n$, let $n_{r^{\prime}}=n / n_{r}$, and let $\mathbf{O}_{r}(G)$ be the largest normal $r$-subgroup of $G$.

Given a group $X$, let $H$ be a core-free subgroup of ( $X$ of) finite index. Take $g$ of $X \backslash H$ such that $g^{2} \in H$, define a coset graph $\Gamma(X, H, g)$ to be the graph with the set of right cosets of $H$ in $X$ as vertex set, and join two vertices $H x$ and $H y$ an edge whenever $x y^{-1} \in H g H$. It is easy to see that $\Gamma(X, H, g)$ has valency $\left|H: H \cap g^{-1} H g\right|$, and it is connected if and only if $\langle H, g\rangle=X$. Moreover, $X$ acts on the right cosets by multiplication induces an arc-transitive subgroup of the automorphism group of $\Gamma(X, H, g)$.

Example 3. Let $X \cong \mathrm{M}_{23}, N \cong \mathrm{C}_{23}$ : $\mathrm{C}_{11}$ be a maximal subgroup of $X$ (see [4]), $H \cong \mathrm{C}_{23}$ be a normal subgroup of $N$ and $g$ be an involution of $X$. As $N$ is the only maximal subgroup of $X$ up to conjugation of order divisible by 23 , it follows that $\langle H, g\rangle=X$ and $N=\mathbf{N}_{X}(H)$. Consequently, $g \notin \mathbf{N}_{X}(H)$ and so $H \cap g^{-1} H g=1$. Thus $\Gamma(X, H, g)$ is a connected $X$-symmetric graph of valency 23. Moreover, $X$ has a subgroup $G \cong \mathrm{M}_{22}$. Since $|G||H|=|X|$ and $\operatorname{gcd}(|G|,|H|)=1$, we see that $G$ acts regularly by right multiplication. Hence $\Gamma(X, H, g)$ is a Cayley graph on $G$. As $G$ is not normal in $X$, this is a non-normal Cayley graph on $G=\mathrm{M}_{22}$.

The following result is well-known (see for example [18, Theorem 1.1]).

Lemma 4. Let $X$ be a transitive permutation group of prime degree $p$. Then one of the following holds:

(a) $\mathrm{C}_{p} \leqslant X \leqslant \operatorname{AGL}(1, p)$;

(b) $X=\mathrm{A}_{p}$ or $\mathrm{S}_{p}$ with $p \geqslant 5$;

(c) $\operatorname{PGL}(d, q) \leqslant X \leqslant \operatorname{P\Gamma L}(d, q)$ and $p=\left(q^{d}-1\right) /(q-1)$, where $d \geqslant 2$ and $q$ is a prime power;

(d) $(X, p)=(\operatorname{PSL}(2,11), 11),\left(\mathrm{M}_{11}, 11\right)$ or $\left(\mathrm{M}_{23}, 23\right)$.

A permutation group $X$ on a set $\Omega$ is said to be quasiprimitive if its non-trivial normal subgroups are all transitive on $\Omega$. For a graph $\Gamma$ and a subgroup $K$ of $\operatorname{Aut}(\Gamma)$, the quotient graph $\Gamma_{K}$ of $\Gamma$ by $K$ is defined to be the graph with vertices the $K$-orbits on $V(\Gamma)$ such that two vertices $\bar{\alpha}$ and $\bar{\beta}$ of $\Gamma_{K}$ are adjacent if and only if there exist $\alpha \in \bar{\alpha}$ and $\beta \in \bar{\beta}$ adjacent in $\Gamma$. 
Proposition 5. ([9, Theorem 1.1]) Let $G$ be a finite non-abelian simple group, $\Gamma=$ Cay $(G, S)$ be a connected Cayley graph on $G$, and $M$ be a subgroup of $\operatorname{Aut}(\Gamma)$ containing $G: \operatorname{Aut}(G, S)$. Then either $M=G$ :Aut $(G, S)$ or one of the following holds:

(a) $M$ is almost simple, and $\operatorname{Soc}(M)>G$ is transitive on $V(\Gamma)$;

(b) $G \cdot \operatorname{Inn}(G) \leqslant M=(G: \operatorname{Aut}(G, S)) \cdot \mathrm{C}_{2}$ and $S$ is a self-inverse union of $G$-conjugacy classes;

(c) $M$ is not quasiprimitive and there is a maximal intransitive normal subgroup $K$ of $M$ such that one of the following holds:

(c.1) $M / K$ is almost simple, and $\operatorname{Soc}(M / K) \geqslant G K / K \cong G$ is transitive on $V\left(\Gamma_{K}\right)$;

(c.2) $M / K=\operatorname{AGL}(3,2), G=\operatorname{PSL}(2,7)$, and $\Gamma_{K}=\mathrm{K}_{8}$;

(c.3) $\operatorname{Soc}(M / K) \cong T \times T$, and $G K / K \cong G$ is a diagonal subgroup of $\operatorname{Soc}(M / K)$, where $T$ and $G$ are given in Table 4 .

Table 4: Product action possibilities

\begin{tabular}{l|l|l|l|l}
\hline & $G$ & $T$ & $m$ & $\left|V\left(\Gamma_{K}\right)\right|$ \\
\hline 1 & $\mathrm{~A}_{6}$ & $G$ & 6 & $m^{2}$ \\
\hline 2 & $\mathrm{M}_{12}$ & $G$ or $\mathrm{A}_{m}$ & 12 & $m^{2}$ \\
\hline 3 & $\mathrm{Sp}_{4}(q)\left(q=2^{a}\right)$ & $G$ or $\mathrm{A}_{m}$ or $\mathrm{Sp}_{4 \mathrm{r}}\left(\mathrm{q}_{0}\right)\left(\mathrm{q}=\mathrm{q}_{0}^{\mathrm{r}}\right)$ & $\frac{q^{2}\left(q^{2}-1\right)}{2}$ & $m^{2}$ \\
\hline 4 & & $\mathrm{Sp}_{4 \mathrm{r}}\left(\mathrm{q}_{0}\right)\left(\mathrm{q}=\mathrm{q}_{0}^{\mathrm{r}}\right)$ & $\frac{q^{2}\left(q^{2}-1\right)}{2}$ & $2 m^{2}$ \\
\hline 5 & $\mathrm{P} \Omega_{8}^{+}(\mathrm{q})$ & $G$ or $\mathrm{A}_{m}$ or $\mathrm{Sp}_{8}(2)($ if $q=2)$ & $\frac{q^{3}\left(q^{4}-1\right)}{(2, q-1)}$ & $m^{2}$ \\
\hline
\end{tabular}

Let $\Gamma$ be a graph, $X \leqslant \operatorname{Aut}(\Gamma)$ and $\{\alpha, \beta\} \in E(\Gamma)$, let $\Gamma(\alpha)$ denote the neighborhood of $\alpha$. Let $X_{\alpha}^{[1]}$ be the kernel of the vertex-stabilizer $X_{\alpha}$ acting on $\Gamma(\alpha)$, and let $X_{\alpha \beta}^{[1]}=$ $X_{\alpha}^{[1]} \cap X_{\beta}^{[1]}$. For a positive integer $s$, an $(s+1)$-sequence $\left(\alpha_{0}, \alpha_{1}, \cdots, \alpha_{s}\right)$ of vertices of $\Gamma$ is called an $s$-arc if $\left\{\alpha_{i-1}, \alpha_{i}\right\} \in E(\Gamma)$ for $i=1, \ldots, s$ and $\alpha_{i-1} \neq \alpha_{i+1}$ for $i=1, \ldots, s-1$. The graph $\Gamma$ is said to be $(X, s)$-arc-transitive if $X$ acts transitively on the set of $s$-arcs of $\Gamma$, and is said to be $(X, s)$-transitive if it is $(X, s)$-arc-transitive but not $(X, s+1)$-arctransitive.

Proposition 6. ([13, Theorem 1.1]) Let $\Gamma$ be a connected $X$-symmetric graph of valency 7. Then for $\alpha \in V(\Gamma), X_{\alpha}$ lies in Table 5 .

The next proposition follows from [12] and [20].

Proposition 7. Let $\Gamma$ be a connected $(X, s)$-transitive graph of prime valency $p>7$ and let $\{\alpha, \beta\}$ be an edge of $\Gamma$. If $X_{\alpha}$ is solvable, then $X_{\alpha} \cong\left(\mathrm{C}_{p}: \mathrm{C}_{m}\right) \times \mathrm{C}_{n}$ for some $m$ dividing $(p-1)$ and $n$ dividing $m$. If $X_{\alpha}$ is nonsolvable, then $\left|X_{\alpha}\right|_{p}=p$, and either $\left(s, p, X_{\alpha}\right)$ lies in Table 6 , or one of the following statements (a)-(c) holds, where $d \geqslant 2$ is an integer and $q=r^{f}$ for some prime $r$ and positive integer $f$ such that $p=\left(q^{d}-1\right) /(q-1)$. 
Table 5:

\begin{tabular}{l|l}
\hline$\left|X_{\alpha}\right|_{2}$ & $X_{\alpha}$ \\
\hline 1 & $\mathrm{C}_{7}, \mathrm{~F}_{21}, \mathrm{~F}_{21} \times \mathrm{C}_{3}$ \\
\hline 2 & $\mathrm{D}_{14}, \mathrm{~F}_{42}, \mathrm{~F}_{42} \times \mathrm{C}_{3}$ \\
\hline $2^{2}$ & $\mathrm{D}_{28}, \mathrm{~F}_{42} \times \mathrm{C}_{2}, \mathrm{~F}_{42} \times \mathrm{C}_{6}$ \\
\hline $2^{3}$ & $\mathrm{SL}(3,2), \mathrm{A}_{7}$ \\
\hline $2^{4}$ & $\mathrm{~S}_{7}$ \\
\hline $2^{6}$ & $\mathrm{C}_{2}^{3}: \mathrm{SL}(3,2), \mathrm{SL}(3,2) \times \mathrm{S}_{4}, \mathrm{~A}_{7} \times \mathrm{A}_{6}$ \\
\hline $2^{7}$ & $\mathrm{C}_{2}^{4}: \mathrm{SL}(3,2),\left(\mathrm{A}_{7} \times \mathrm{A}_{6}\right): \mathrm{C}_{2}$ \\
\hline $2^{8}$ & $\mathrm{~S}_{6} \times \mathrm{S}_{7}$ \\
\hline $2^{10}$ & $\mathrm{C}_{2}^{6}:\left(\mathrm{SL}(3,2) \times \mathrm{S}_{3}\right)$ \\
\hline $2^{24}$ & {$\left[2^{20}:\left(\mathrm{SL}(3,2) \times \mathrm{S}_{3}\right)\right.$} \\
\hline
\end{tabular}

Table 6:

\begin{tabular}{l|l|l}
\hline$s$ & $p$ & $X_{\alpha}$ \\
\hline 2 & $p$ & $\mathrm{~A}_{p}, \mathrm{~S}_{p}$ \\
\hline 2 & 11 & $\mathrm{PSL}(2,11), \mathrm{M}_{11}$ \\
\hline 2 & 23 & $\mathrm{M}_{23}$ \\
\hline 3 & $p$ & $\mathrm{~A}_{p-1} \times \mathrm{A}_{p},\left(\mathrm{~A}_{p-1} \times \mathrm{A}_{p}\right): \mathrm{C}_{2}, \mathrm{~S}_{p-1} \times \mathrm{S}_{p}$ \\
\hline 3 & 11 & $\mathrm{~A}_{5} \times \mathrm{PSL}(2,11), \mathrm{A}_{6} \times \mathrm{M}_{11}, \mathrm{M}_{10} \times \mathrm{M}_{11}$ \\
\hline 3 & 23 & $\mathrm{M}_{22} \times \mathrm{M}_{23}$ \\
\hline
\end{tabular}

(a) $s=2$ and one of the following holds:

(a.1) $d=2, r=2, \operatorname{PSL}(2, q) \leqslant X_{\alpha} \leqslant \operatorname{P\Gamma L}(2, q)$ and $X_{\alpha \beta}^{[1]}=1$;

(a.2) $d \geqslant 3, X_{\alpha}=\left(\left(\mathrm{C}_{r}^{f(d-1)}: \mathrm{C}_{\ell}\right) \times \operatorname{PSL}(d, q)\right) \cdot \mathcal{O}$ and $X_{\alpha \beta}^{[1]}=1$, where $\mathcal{O} \leqslant \mathrm{C}_{f}$ and $\mathrm{C}_{\ell} \leqslant \mathrm{C}_{q-1}$

(a.3) $d \geqslant 3, X_{\alpha}=\mathrm{O}_{r}\left(X_{\alpha}\right) \cdot \mathrm{C}_{\ell} \cdot \operatorname{PSL}(d, q) \cdot \mathcal{O}$ and $X_{\alpha \beta}^{[1]} \neq 1$, where $\mathcal{O} \leqslant \mathrm{C}_{f}$ and $\mathrm{C}_{\ell} \leqslant$ $\mathrm{C}_{q-1}$.

(b) $s=3$ and one of the following holds:

(b.1) $d=2, r=2, X_{\alpha}=\left(\left(\mathrm{C}_{2}^{f} \cdot \mathcal{O}_{1}\right) \times \operatorname{PSL}(2, q)\right) \cdot \mathcal{O}$ and $X_{\alpha \beta}^{[1]}=1$, where $\mathcal{O} \leqslant \mathrm{C}_{f}$ and $\mathcal{O}_{1} \leqslant \mathrm{C}_{q-1} \cdot \mathcal{O}$

(b.2) $d \geqslant 3, X_{\alpha}=\left(\left(\mathrm{C}_{r}^{f(d-1)}: \mathrm{C}_{\ell} \cdot \operatorname{PSL}(d-1, q) \cdot \mathcal{O}^{\prime}\right) \times \operatorname{PSL}(d, q)\right) \cdot \mathcal{O}$ and $X_{\alpha \beta}^{[1]}=1$, where $\mathcal{O} \leqslant \mathrm{C}_{f}, \mathrm{C}_{\ell} \leqslant \mathrm{C}_{q-1}$ and $\mathcal{O}^{\prime} \leqslant \mathrm{C}_{\operatorname{gcd}(d-1, q-1)} \cdot \mathcal{O}$; 
(b.3) $d \geqslant 3, X_{\alpha}=\mathrm{O}_{r}\left(X_{\alpha}\right) \cdot \mathrm{C}_{\ell} \cdot\left(\left(\operatorname{PSL}(d-1, q) \cdot \mathcal{O}^{\prime}\right) \times \operatorname{PSL}(d, q)\right) \cdot \mathcal{O}$ and $X_{\alpha \beta}^{[1]} \neq 1$, where $\mathcal{O} \leqslant \mathrm{C}_{f}, \mathrm{C}_{\ell} \leqslant \mathrm{C}_{q-1}$ and $\mathcal{O}^{\prime} \leqslant \mathrm{C}_{\operatorname{gcd}(d-1, q-1)} \cdot \mathcal{O}$; moreover, if $r \geqslant 5$ then $\left|\mathbf{O}_{r}\left(X_{\alpha}\right)\right|$ divides $q^{d(d-1)}$.

(c) $s=5, d=2, r=2, X_{\alpha}=\left(\left[q^{3}\right]: \mathrm{GL}(2, q)\right) \cdot \mathcal{O}$ and $X_{\alpha \beta}^{[1]}=1$, where $\mathcal{O} \leqslant \mathrm{C}_{f}$.

Recall that a permutation group is called $k$-homogeneous if it is transitive on the $k$ sets of permuted points. The following result is about the $k$-homogeneous groups which can be get from [15, Theorem 1].

Lemma 8. Let $G$ be a group $k$-homogeneous but not $k$-transitive on a finite set $\Omega$ of $n$ points, where $n \geqslant 2 k$. Then, up to permutation isomorphism, one of the following holds:

(a) $k=2$ and $G \leqslant \operatorname{A\Gamma L}(1, q)$ with $n=q \equiv 3 \bmod 4$;

(b) $k=3$ and $\operatorname{PSL}(2, q) \leqslant G \leqslant \operatorname{P\Gamma L}(2, \mathrm{q})$, where $n-1=q \equiv 3 \bmod 4$;

(c) $k=3$ and $G=\operatorname{AGL}(1,8), \operatorname{A\Gamma L}(1,8)$ or $\mathrm{A} \Gamma \mathrm{L}(1,32)$;

(d) $k=4$ and $G=\operatorname{PSL}(2,8), \operatorname{P\Gamma L}(2,8)$ or $\operatorname{P\Gamma L}(2,32)$.

\section{Proof of the main result}

In the following section, we give the proof of our main theorem.

Lemma 9. Let $X$ be a permutation group on a set $\Omega$, let $G$ be a transitive subgroup of $X$. Let $\alpha \in \Omega$, suppose that both $X$ and $G$ are non-abelian simple and $X_{\alpha}$ is as described in Proposition 6 or 7 . Then either $X$ is a classical simple group or $\left(X, G, X_{\alpha}\right)$ lies in Table 7.

Proof. From Propositions 6 and 7 we see that there exists a prime $p \geqslant 7$ such that $\left|X_{\alpha}\right|_{p}=p$. As $G$ is transitive, we have $X=G X_{\alpha}$. Suppose that $X$ is not a classical simple group. Then $X$ is an alternating group or a simple group of exceptional Lie type or a sporadic simple group.

First assume that $X$ is a simple group of exceptional Lie type. Since $X=G X_{\alpha}$ with $G$ non-abelian simple, it follows from [14, Theorem 1] that $\left(X, G, X_{\alpha}\right)$ lies in Table 8. In line 1 of Table $8, X_{\alpha}$ has a composition factor $\operatorname{PSU}(3,4)$, which is not as described in Proposition 6 or 7 , a contradiction. Similarly one may exclude lines 2 and 6-8 of Table 8 . For the line 4 or $5, X_{\alpha}$ has a composition factor $\operatorname{PSL}(3, q)$ with $q$ a 3 -power, and has no non-trivial solvable normal subgroup. It can be seen that only cases (a.2)-(a.3) and (b.2)(b.3) of Proposition 7 satisfy that $X_{\alpha}$ has a composition factor $\operatorname{PSL}(3, q)$. However, in those cases $X_{\alpha}$ has a non-trivial solvable normal subgroup, a contradiction. Similarly one may exclude line 3 of Table 8 . Hence, none of the triples $\left(X, G, X_{\alpha}\right)$ in Table 8 happens.

Next, assume that $X$ is a sporadic simple group. By [11, Theorem 1.1], we know that $\left(X, G, X_{\alpha}\right)$ lies in Table 9. As $X_{\alpha}$ is described in Proposition 6 or 7 , thus $\left(X, G, X_{\alpha}\right)$ 
Table 7:

\begin{tabular}{|c|c|c|c|c|}
\hline & $X$ & $G$ & $X_{\alpha}$ & conditions \\
\hline 1 & $\mathrm{~A}_{n}$ & $\mathrm{~A}_{n-1}$ & $\begin{array}{l}\text { transitive permutation } \\
\text { group of degree } n\end{array}$ & $n \geqslant 6$ \\
\hline 2 & $\mathrm{~A}_{p}$ & $\mathrm{~A}_{p-2}$ & $\operatorname{PGL}(d, q) \cdot\langle\sigma\rangle$ & $p=\frac{q^{d}-1}{q-1}, \sigma \mid f$ \\
\hline 3 & $\mathrm{~A}_{p}$ & $\mathrm{~A}_{p-3}$ & $\begin{array}{l}\operatorname{PGL}(2, q) \cdot\langle\sigma\rangle \\
\operatorname{AGL}(d, 2)\end{array}$ & $\begin{array}{c}p=q+1, \sigma \mid f \\
p=2^{d}-1, d \text { odd }\end{array}$ \\
\hline 4 & $\mathrm{~A}_{11}$ & $\begin{array}{l}A_{9} \\
A_{7}\end{array}$ & $\begin{array}{c}\operatorname{PSL}(2,11) \\
\mathrm{M}_{11}\end{array}$ & $\begin{array}{l}p=11 \\
p=11\end{array}$ \\
\hline 5 & $\mathrm{~A}_{23}$ & $\mathrm{~A}_{19}$ & $\mathrm{M}_{23}$ & $p=23$ \\
\hline 6 & $\mathrm{~A}_{p+1}$ & $\begin{array}{l}\text { transitive permutation } \\
\text { group of degree } p+1\end{array}$ & $\mathrm{~A}_{p}$ & $p$ prime \\
\hline 7 & $\mathrm{~A}_{p+3}$ & $\operatorname{PSL}(2, p+2)$ & $\mathrm{S}_{p}$ & $p \equiv 1 \quad(\bmod 4)$ \\
\hline 8 & $\begin{array}{l}\mathrm{A}_{11} \\
\mathrm{~A}_{12}\end{array}$ & $\begin{array}{l}\mathrm{M}_{11} \\
\mathrm{M}_{12}\end{array}$ & $\mathrm{~A}_{7}$ or $\mathrm{S}_{7}$ & $p=7$ \\
\hline 9 & $\begin{array}{l}\mathrm{A}_{23} \\
\mathrm{~A}_{24}\end{array}$ & $\begin{array}{l}M_{23} \\
M_{24}\end{array}$ & $\mathrm{~A}_{19}$ or $\mathrm{S}_{19}$ & $p=19$ \\
\hline 10 & $\mathrm{~A}_{p+k}$ & $\operatorname{PSL}(d, q)$ & $\mathrm{A}_{p}$ or $\mathrm{S}_{p}$ & $\frac{q^{d}-1}{q-1}=p+k, k=2$ or 3 \\
\hline 11 & $\mathrm{~A}_{8}$ & $\begin{array}{l}\mathrm{A}_{5} \\
\mathrm{~A}_{k}\end{array}$ & $\begin{array}{c}\operatorname{AGL}(3,2) \\
\operatorname{SL}(3,2), \operatorname{AGL}(3,2)\end{array}$ & $\begin{array}{c}p=7 \\
p=7, k \in\{6,7\}\end{array}$ \\
\hline 12 & $\mathrm{M}_{12}$ & $\mathrm{M}_{11}$ & $\mathrm{M}_{11}, \operatorname{PSL}(2,11)$ & $p=11$ \\
\hline 13 & $\mathrm{M}_{12}$ & $\operatorname{PSL}(2,11)$ & $\mathrm{M}_{11}$ & $p=11$ \\
\hline 14 & $\mathrm{M}_{12}$ & $\mathrm{~A}_{5}$ & $\mathrm{M}_{11}$ & $p=11$ \\
\hline 15 & $\mathrm{M}_{23}$ & $\mathrm{M}_{22}$ & $\mathrm{C}_{23}, \mathrm{C}_{23}: \mathrm{C}_{11}$ & $p=23$ \\
\hline 16 & $\mathrm{M}_{24}$ & $\mathrm{M}_{23}$ & $\mathrm{SL}(3,2), \mathrm{C}_{2}^{6}:\left(\mathrm{SL}(3,2) \times \mathrm{S}_{3}\right)$ & $p=7$ \\
\hline 17 & $\mathrm{M}_{24}$ & $\operatorname{PSL}(2,23)$ & $\mathrm{M}_{23}$ & $p=23$ \\
\hline
\end{tabular}


Table 8:

\begin{tabular}{l|l|l|l}
\hline & $X$ & $G$ & $X_{\alpha}$ \\
\hline 1 & $\mathrm{G}_{2}(4)$ & $\mathrm{J}_{2}$ & $\operatorname{PSU}(3,4), \operatorname{PSU}(3,4) \cdot \mathrm{C}_{2}$ \\
\hline 2 & $\mathrm{G}_{2}(4)$ & $\operatorname{PSU}(3,4)$ & $\mathrm{J}_{2}$ \\
\hline 3 & $\mathrm{G}_{2}\left(3^{f}\right)$ & $\operatorname{PSL}\left(3,3^{f}\right)$ & $\operatorname{PSU}\left(3,3^{f}\right), \operatorname{PSU}\left(3,3^{f}\right) \cdot \mathrm{C}_{2}$ \\
\hline 4 & $\mathrm{G}_{2}\left(3^{f}\right)$ & $\operatorname{PSU}\left(3,3^{f}\right)$ & $\operatorname{PSL}\left(3,3^{f}\right), \operatorname{PSL}\left(3,3^{f}\right) \cdot \mathrm{C}_{2}$ \\
\hline 5 & $\mathrm{G}_{2}\left(3^{2 e+1}\right)$ & ${ }^{2} G_{2}\left(3^{2 e+1}\right)$ & $\operatorname{PSL}\left(3,3^{2 e+1}\right), \operatorname{PSL}\left(3,3^{2 e+1}\right) \cdot \mathrm{C}_{2}$ \\
\hline 6 & $\mathrm{G}_{2}\left(3^{2 e+1}\right)$ & $\operatorname{PSL}\left(3,3^{2 e+1}\right)$ & ${ }^{2} G_{2}\left(3^{2 e+1}\right)$ \\
\hline 7 & $\mathrm{~F}_{4}\left(2^{f}\right)$ & $\operatorname{Sp}\left(8,2^{f}\right)$ & ${ }^{3} \mathrm{D}_{4}\left(2^{f}\right),{ }^{3} \mathrm{D}_{4}\left(2^{f}\right) \cdot \mathrm{C}_{3}$ \\
\hline 8 & $\mathrm{~F}_{4}\left(2^{f}\right)$ & ${ }^{3} \mathrm{D}_{4}\left(2^{f}\right)$ & $\operatorname{Sp}\left(8,2^{f}\right)$ \\
\hline
\end{tabular}

Table 9:

\begin{tabular}{|c|c|c|c|}
\hline & $X$ & G & $X_{\alpha}$ \\
\hline 1 & $\mathrm{M}_{12}$ & $\mathrm{M}_{11}$ & $\mathrm{M}_{11}, \operatorname{PSL}(2,11)$ \\
\hline 2 & $\mathrm{M}_{12}$ & $\operatorname{PSL}(2,11)$ & $\mathrm{M}_{11}$ \\
\hline 3 & $\mathrm{M}_{12}$ & $\mathrm{~A}_{5}$ & $\mathrm{M}_{11}$ \\
\hline 4 & $\mathrm{M}_{23}$ & $\mathrm{M}_{22}$ & $\mathrm{C}_{23}, \mathrm{C}_{23}: \mathrm{C}_{11}$ \\
\hline 5 & $\mathrm{M}_{24}$ & $\mathrm{M}_{23}$ & $\begin{array}{l}\mathrm{M}_{12} \cdot \mathrm{C}_{2}, \mathrm{C}_{2}^{3}: \mathrm{F}_{21}, \mathrm{C}_{2}^{6}: \mathrm{C}_{21}, \mathrm{C}_{2}^{6}: \mathrm{F}_{21}, \mathrm{C}_{2}^{6}: \mathrm{C}_{7}: \mathrm{S}_{3}, \mathrm{C}_{2}^{6}:\left(\mathrm{F}_{21} \times \mathrm{C}_{3}\right), \\
\mathrm{C}_{2}^{6}:\left(\mathrm{F}_{21} \times \mathrm{S}_{3}\right), \mathrm{C}_{2}^{6}:\left(\mathrm{SL}(3,2) \times \mathrm{C}_{3}\right), \mathrm{C}_{2}^{6}:\left(\mathrm{SL}(3,2) \times \mathrm{S}_{3}\right), \\
\mathrm{SL}(3,2), \mathrm{SL}(3,2) \times \mathrm{C}_{3}, \mathrm{SL}(3,2) \times \mathrm{S}_{3}, \mathrm{PGL}(2,11), \operatorname{PSL}(2,23)\end{array}$ \\
\hline 6 & $\mathrm{M}_{24}$ & $\operatorname{PSL}(2,23)$ & $\mathrm{P} \Sigma \mathrm{L}(3,4), \mathrm{PSL}(3,4) . \mathrm{S}_{3}, \mathrm{C}_{2}^{4}: \mathrm{A}_{7}, \mathrm{C}_{2}^{4}: \mathrm{A}_{8}, \mathrm{M}_{22} . \mathrm{C}_{2}, \mathrm{M}_{22}, \mathrm{M}_{23}$ \\
\hline 7 & $\mathrm{M}_{24}$ & $\operatorname{PSL}(2,7)$ & $\mathrm{M}_{23}$ \\
\hline 8 & $\mathrm{HS}$ & $\mathrm{M}_{22}$ & $\mathrm{PSU}(3,5) \cdot \mathrm{C}_{2}$ \\
\hline 9 & $\mathrm{Ru}$ & $\operatorname{PSL}(2,29)$ & ${ }^{2} \mathrm{~F}_{4}(2)$ \\
\hline 10 & Suz & $\mathrm{G}_{2}(4)$ & $\operatorname{PSU}(5,2), \mathrm{C}_{3}^{5}: \mathrm{M}_{11}$ \\
\hline 11 & Suz & $\mathrm{PSU}(5,2)$ & $\mathrm{G}_{2}(4)$ \\
\hline 12 & $\mathrm{Fi}_{22}$ & ${ }^{2} \mathrm{~F}_{4}(2)^{\prime}$ & $\mathrm{C}_{2} \cdot \mathrm{PSU}(6,2)$ \\
\hline 13 & $\mathrm{Co}_{1}$ & $\mathrm{Co}_{2}$ & $\left(\mathrm{C}_{3} \cdot \mathrm{Suz}\right) \cdot \mathrm{C}_{2}, \mathrm{C}_{3} \cdot \mathrm{Suz}$ \\
\hline 14 & $\mathrm{Co}_{1}$ & $\mathrm{CO}_{2}$ & $\mathrm{G}_{2}(4) \leqslant X_{\alpha} \leqslant\left(\mathrm{A}_{4} \times \mathrm{G}_{2}(4)\right) \cdot \mathrm{C}_{2}$ \\
\hline 15 & $\mathrm{Co}_{1}$ & $\mathrm{G}_{2}(4)$ & $\mathrm{Co}_{2}$ \\
\hline 16 & $\mathrm{Co}_{1}$ & $\mathrm{Co}_{3}$ & $\left(\mathrm{C}_{3} . \mathrm{Suz}\right) \cdot \mathrm{C}_{2}, \mathrm{C}_{3} . \mathrm{Suz}$ \\
\hline 17 & $\mathrm{Co}_{1}$ & $\mathrm{Co}_{3}$ & $\mathrm{G}_{2}(4) \cdot \mathrm{C}_{2} \leqslant X_{\alpha} \leqslant\left(\mathrm{A}_{4} \times \mathrm{G}_{2}(4)\right) \cdot \mathrm{C}_{2}$ \\
\hline
\end{tabular}


cannot be lines 8-17 of Table 9 . If $\left(X, G, X_{\alpha}\right)$ lies in lines 1-4 of Table 9 , then one of lines $12-15$ of Table 7 holds. If $\left(X, G, X_{\alpha}\right)$ lies in line $5-6$ of Table 9 , then $p=7,11$ or 23. Furthermore, by Proposition 6 and 7 , we have lines 16-17 of Table 7 hold. If $\left(X, G, X_{\alpha}\right)$ lies in line 7 of Table 9 , then $\left(X, G, X_{\alpha}\right) \cong\left(\mathrm{M}_{24}, \operatorname{PSL}(2,27), \mathrm{M}_{23}\right)$. Note that $\left|G \cap X_{\alpha}\right|=\frac{|\operatorname{PSL}(2,7)|\left|\mathrm{M}_{23}\right|}{\left|\mathrm{M}_{24}\right|}=7$. It follows that $X_{\alpha} \cong \mathrm{M}_{23}$, which has no subgroup of index 7, a contradiction. Hence this case cannot happen.

Finally, let $X$ be the alternating group $\mathrm{A}_{n}$ naturally acts on a set $\Theta$ of $n$ points with $n \geqslant 5$. Again, as $X=G X_{\alpha}$, we derive from [22, Theorem D and Remark 2] (which gave the maximal factorizations of the alternating groups) that one of the following holds:

(i) $G=\mathrm{A}_{n-k}$ for some $1 \leqslant k \leqslant 5$ and $X_{\alpha}$ is $k$-homogenous on $\Theta$;

(ii) $G$ is $k$-homogenous on $\Theta$ and $\mathrm{A}_{n-k} \unlhd X_{\alpha} \leqslant\left(\mathrm{S}_{n-k} \times \mathrm{S}_{k}\right) \cap \mathrm{A}_{n}$ for some $1 \leqslant k \leqslant 5$;

(iii) $n=6, G=\operatorname{PSL}(2,5), X_{\alpha} \leqslant \mathrm{S}_{3} 2 \mathrm{~S}_{2}$ and $X_{\alpha}$ is transitive on $\Theta$;

(iv) $n=10, G=\operatorname{PSL}(2,8), \mathrm{A}_{5} \times \mathrm{A}_{5} \unlhd X_{\alpha} \leqslant \mathrm{S}_{5} \prec \mathrm{S}_{2}$ and $X_{\alpha}$ is transitive on $\Theta$.

To finish the proof, in the following, we analyze the above four cases (i)-(iv) one by one.

Case (i). Suppose that $G=\mathrm{A}_{n-k}$ for some $1 \leqslant k \leqslant 5$ and $X_{\alpha}$ is $k$-homogenous on $\Theta$. If $k=1$, then $n \geqslant 6$ and $X_{\alpha}$ is transitive on $\Theta$, as in line 1 of Table 7 . Henceforth assume $k \geqslant 2$. Since $G=\mathrm{A}_{n-k}$ is non-abelian simple group, $n-k \geqslant 5$, i.e., $n \geqslant 5+k$. Note that, if $1 \leqslant k \leqslant 5$, then $n \geqslant 2 k$.

Assume that $X_{\alpha}$ is $k$-homogeneous but not $k$-transitive. Then $X_{\alpha}$ is one of the four cases in Lemma 8 , and especially we have $k \leqslant 4$. In the following, we will analyze these four cases one by one. Note that we have $|X: G|=\left|\mathrm{A}_{n}: \mathrm{A}_{n-k}\right|=n(n-1) \cdots(n-k+1)$ and $|X: G||| X_{\alpha} \mid$.

Let $q=r^{f}$ for some prime $r$ and positive integer $f$. If $k=2$, then $X_{\alpha} \leqslant \operatorname{A\Gamma L}(1, q)$ with $n=q \equiv 3 \bmod 4$. Note that $G \cong \mathrm{A}_{q-2},|X: G||| X_{\alpha} \mid$ with $|X: G|=q(q-1)$ and $X_{\alpha} \leqslant A \Gamma L(1, q) \cong \mathrm{C}_{r}^{f}:\left(\mathrm{C}_{q-1}: \mathrm{C}_{f}\right)$ for $q=r^{f}$. It follows that $X_{\alpha} \cong \mathrm{C}_{r}^{f}:\left(\mathrm{C}_{q-1}: \mathrm{C}_{\ell}\right)$ for $\ell \mid f$, and so $X_{\alpha}$ is 2-transitive on $\Omega$, a contradiction. Suppose that $k=3$. Then $G \cong \mathrm{A}_{n-3}$ for $n \geqslant 8$, and $|X: G|=n(n-1)(n-2)$ is a factor of $\left|X_{\alpha}\right|$. On the other hand, Lemma 8 shows that either $\operatorname{PSL}(2, q) \leqslant X_{\alpha} \leqslant \operatorname{P\Gamma L}(2, q)$ with $n-1=q \equiv 3 \bmod 4$, or $X_{\alpha}=\operatorname{AGL}(1,8), \operatorname{A\Gamma L}(1,8)$ or $\operatorname{A\Gamma L}(1,32)$. For the latter case, a calculation of the order for these candidates of $X_{\alpha}$ shows that this case cannot occur. Suppose that the former case occurs. Then $n=q+1, n(n-1)(n-2)=(q-1) q(q+1)$ is a factor of $\left|X_{\alpha}\right|$, and $X_{\alpha} \cong \operatorname{PSL}(2, q) \cdot\left(C_{2} \times \mathrm{C}_{l}\right)$ for $l \mid f$ (see ). Upon to Lemma $7, p=\frac{q^{2}-1}{q-1}=q+1$, and so $n=p$. It is clear that $X_{\alpha}$ is 2-transitive on $\Theta=\{1, \cdots, p\}$, a contradiction. Assume that $k=4$. Then $G \cong \mathrm{A}_{n-4}$ for $n \geqslant 9$ and $\left|X: X_{\alpha}\right|=n(n-1)(n-2)(n-3)$ is a factor of $\left|X_{\alpha}\right|$. In particular, Lemma 8 shows that $X_{\alpha} \cong \operatorname{PSL}(2,8), \operatorname{P\Gamma L}(2,8)$ or $\operatorname{P\Gamma L}(2,32)$. A calculation of the orders for those candidates of $X_{\alpha}$ shows that this case cannot occur.

Now we suppose that $X_{\alpha}$ is $k$-transitive on $\Theta$ for $k \geqslant 2$. Note that $\left(\mathrm{C}_{p}: \mathrm{C}_{p-1}\right): \mathrm{C}_{\ell}$ with $n=p \geqslant 7$ prime and $\ell \mid(p-1)$, is not isomorphic to a subgroup of $\mathrm{A}_{p}$ as $\mathrm{C}_{p-1}$ contains an element of odd permutation. Then since $X_{\alpha}$ is a $k$-transitive subgroup of $\mathrm{A}_{n}$ and is also as described in Proposition 6 or 7 , one can get that either $\operatorname{PSL}(d, q) \leqslant X_{\alpha} \leqslant \operatorname{P\Gamma L}(d, q)$ 
with $n=p=\left(q^{d}-1\right) /(q-1) \geqslant 7$ prime for some integer $d \geqslant 2$ and prime power $q$, or $\left(X_{\alpha}, n\right)=(\operatorname{PSL}(2,11), 11),\left(\mathrm{M}_{11}, 11\right)$ or $\left(\mathrm{M}_{23}, 23\right)$. For the latter case, one can deduce that line 4-5 of Table 7 hold. Now assume that the former case occurs. Then since $X_{\alpha}$ is a $k$-transitive permutation group for $k \geqslant 2$, by [3, Theorem 4.11], we have $k \leqslant 3$. Again, as $X_{\alpha}$ is described in Proposition 6 or 7 , we deduce that if $k=2$, then line 2 of Table 7 holds. For $k=3, X_{\alpha}$ is a 3-transitive permutation group, and so $X_{\alpha} \cong \operatorname{PGL}(2, q) \cdot\langle\sigma\rangle$ for $p=q+1$ and $\sigma \mid f$, or AGL $(d, 2)$ for $p=2^{d}-1$ (see [3, Table 7.3, 7.4] for example). For $p=2^{d}-1$ is prime, then $d$ is odd. Hence line 3 of Table 7 holds.

Case (ii). Assume that $G$ is $k$-homogenous on $\Theta$ and $\mathrm{A}_{n-k} \unlhd X_{\alpha} \leqslant\left(\mathrm{S}_{n-k} \times \mathrm{S}_{k}\right) \cap \mathrm{A}_{n}$ for some $1 \leqslant k \leqslant 5$. Note that $X_{\alpha}$ is given in Proposition 6 or 7 . Then $X_{\alpha} \cong \mathrm{A}_{n-k}$ or $\mathrm{S}_{n-k}$ for $1 \leqslant k \leqslant 5$ and $n-k=p$. If $k=1$, then $\left(X, X_{\alpha}\right) \cong\left(\mathrm{A}_{p+1}, \mathrm{~A}_{p}\right)$ and $G$ is a transitive permutation group of degree $n=p+1$. Hence the line 6 of Table 7 holds.

For $k \geqslant 2$, assume that $G$ is $k$-homogeneous but not $k$-transitive, then $G$ is given in Lemma 8. Let $q=r^{f}$ for some prime $r$ and positive integer $f$. Note that $n \geqslant 5+k$ and $\left|X: X_{\alpha}\right|=n(n-1) \cdots(n-k+1)$ or $\frac{n(n-1) \cdots(n-k+1)}{2}$ respecting to $X_{\alpha} \cong \mathrm{A}_{n-k}$ or $\mathrm{S}_{n-k}$ for $n-k=p$. Since $\left|X: X_{\alpha}\right||| G \mid$ and $G$ is $k$-homogeneous but not $k$-transitive, by a careful analysis of the cases (a)-(d) in Lemma 8, we can draw that $k=3$ and $G \cong \operatorname{PSL}(2, q)$ with $n-1=q \equiv 3 \bmod 4$. Then $p=n-3$ and $q=n-1$ is odd, and so $n$ is even. Therefore, $|G|=|\operatorname{PSL}(2, q)|=\frac{q(q-1)(q+1)}{(2, q-1)}=\frac{n(n-1)(n-2)}{(2, n-2)}=\frac{n(n-1)(n-2)}{2}$. Furthermore, since $|X: G||| X_{\alpha} \mid$, we conclude that $X_{\alpha} \cong \mathrm{S}_{p}$. We derive from $q=n-1$ and $p=n-3$ that $q=p+2$. It follows that $p \equiv 1 \bmod 4$ as $q \equiv 3 \bmod 4$. Hence the line 7 of Table 7 holds.

Now suppose that $G$ is $k$-transitive on $\Theta$. Note that $X_{\alpha} \cong \mathrm{A}_{n-k}$ or $\mathrm{S}_{n-k}$ with $n-k=p$. Since $G<X \cong \mathrm{A}_{n}$ is a non-abelian simple group and $k \geqslant 2$, by [3, Theorem 4.11], we conclude that $2 \leqslant k \leqslant 5$, and if $k=4$ or 5 , then $(G, n, k)=\left(\mathrm{M}_{11}, 11,4\right),\left(\mathrm{M}_{12}, 12,5\right)$, $\left(\mathrm{M}_{23}, 23,4\right)$ or $\left(\mathrm{M}_{24}, 24,5\right)$. It follows that $(X, G, p)=\left(\mathrm{A}_{11}, \mathrm{M}_{11}, 7\right),\left(\mathrm{A}_{12}, \mathrm{M}_{12}, 7\right),\left(\mathrm{A}_{23}\right.$, $\left.\mathrm{M}_{23}, 19\right)$ or $\left(\mathrm{A}_{24}, \mathrm{M}_{24}, 19\right)$ respectively, and hence lines 8-9 of Table 7 hold. Now for $k=2$ or 3. Since $G$ is non-abelian simple, $G$ is given in [3, Table 7.4]. Together with the conditions that $\left|X: X_{\alpha}\right||| G \mid$ and $X_{\alpha} \cong \mathrm{A}_{n-k}$ or $\mathrm{S}_{n-k}$ for $n-k=p$, we can deduce that either $G \cong \operatorname{PSL}(d, q)$ for $n=\left(q^{d}-1\right) /(q-1), d \geqslant 2$ and $q$ being a prime power, or $G \cong$ $\operatorname{Sp}(2 d, 2)$ for $n=2^{2 d-1} \pm 2^{d-1}$ and $d \geqslant 3$. For the latter case, we derive from $n-k=p \geqslant 7$ is an odd prime that $2^{2 d-1} \pm 2^{d-1}-2=p$ or $2^{2 d-1} \pm 2^{d-1}-3=p$. However, $2^{2 d-1} \pm 2^{d-1}-2$ is even, which leads to that $2^{2 d-1} \pm 2^{d-1}-3=p$. Noting that $2^{2 d-1}+2^{d-1}-3=2^{2 d-1}-2+$ $2^{d-1}-1=2\left(\left(2^{d-1}\right)^{2}-1\right)+2^{d-1}-1=2\left(2^{d-1}-1\right)\left(2^{d-1}+1\right)+2^{d-1}-1=\left(2^{d-1}-1\right)\left(2\left(2^{d-1}+1\right)+1\right)$ is not a prime, we conclude that this case cannot occur. Then along the same lines as the previous case, we see that $2^{2 d-1}-2^{d-1}-3=\left(2^{d-1}+1\right)\left(2\left(2^{d-1}-1\right)-1\right)$ is also not prime. It yields that $G \supsetneqq \operatorname{Sp}(2 d, 2)$ for $n=2^{2 d-1} \pm 2^{d-1}$ and $d \geqslant 3$, and hence line 10 of Table 7 holds.

Cases (iii) and (iv). Suppose that $n=6, G=\operatorname{PSL}(2,5), X_{\alpha} \leqslant \mathrm{S}_{3} 2 \mathrm{~S}_{2}$ and $X_{\alpha}$ is transitive on $\Omega$; or $n=10, G=\operatorname{PSL}(2,8), \mathrm{A}_{5} \times \mathrm{A}_{5} \unlhd X_{\alpha} \leqslant \mathrm{S}_{5} \prec \mathrm{S}_{2}$ and $X_{\alpha}$ is transitive on $\Omega$. Since $X_{\alpha}$ is given in Proposition 6 or 7 , in particular, $\left|X_{\alpha}\right|_{p}=p \geqslant 7$, we can deduce that those cases cannot occur. 
In the rest of this section, we always let $G$ be a finite non-abelian simple group, let $\Gamma=\operatorname{Cay}(G, S)$ be a connected symmetric Cayley graph on $G$ of prime valency $p \geqslant 7$, and let $L=\operatorname{Soc}($ Aut $\Gamma)$ and $\alpha$ be a vertex of $\Gamma$. Moreover, for short, let $A=\operatorname{Aut} \Gamma$. If $A=$ $G$.Aut $(G, S)$, then $\Gamma$ is a normal Cayley graph. Now we assume that $A>G$. Aut $(G, S)$.

Lemma 10. Assume that $A$ acts quasiprimitively on $V(\Gamma)$. Then either $L$ is a classical simple group or $\Gamma$ is isomorphic to one of the lines of Table 1.

Proof. Since $A$ is quasiprimitive on $V(\Gamma)$, then either $(a)$ or $(b)$ of Proposition 5 occurs.

Case (i). Suppose that (b) holds. Then the action of $G$ on $S$ by conjugation is either trivial or faithful as $G$ is simple. If the action is trivial, then $G$ is abelian as $S$ generates $G$, a contradiction. Suppose that the action is faithful. Note that $\operatorname{Inn}(G) \unlhd \operatorname{Aut}(G)$ and $S^{\operatorname{Inn}(G)}=S$. Then $\operatorname{Inn}(G) \unlhd \operatorname{Aut}(G, S)$. Since $A=(G$.Aut $(G, S)) \cdot \mathrm{C}_{2}$ and $|S|$ is odd prime, then $\operatorname{Aut}(G, S)$ acts transitively on $S$, and so it is primitive. It follows that $\operatorname{Inn}(G)$ is a transitive permutation group of degree $p$, so does $G$ as $G \cong \operatorname{Inn}(G)$. Further, by Lemma $4, G \cong \operatorname{PSL}(2,11)$ for $p=11, \mathrm{M}_{11}$ for $p=11$ or $\mathrm{M}_{23}$ for $p=23, \mathrm{~A}_{p}, \operatorname{PSL}(d, q)$ for $p=\left(q^{d}-1\right) /(q-1)$, where $d \geqslant 2$ and $q$ is a prime power. On the other hand, since $G \cdot \operatorname{Inn}(G) \leqslant A=(G \cdot \operatorname{Aut}(G, S)) \cdot \mathrm{C}_{2},|A|=|G|\left|A_{1}\right|$ and $\operatorname{Aut}(G, S) \leqslant A_{1}$ for identity $1 \in G$ a vertex of $\Gamma$, then $\left|A_{1}\right|=2|\operatorname{Aut}(G, S)|$ and $A_{\alpha} \cong A_{1} \cong \operatorname{Aut}(G, S) \cdot \mathrm{C}_{2}$.

Assume that $G \cong \operatorname{PSL}(2,11)$ for $p=11$. Then $\operatorname{Inn}(G) \cong G \cong \operatorname{PSL}(2,11)$ and $\operatorname{Aut}(G, S) \cong \operatorname{PSL}(2,11)$ or $\operatorname{PSL}(2,11) \cdot \mathrm{C}_{2}$. Since $A_{1} \cong \operatorname{Aut}(G, S) \cdot \mathrm{C}_{2}$, by Proposition $7(\mathrm{~b})$, we have $A_{1} \cong \operatorname{PSL}(2,11)$, and so $A_{1}=\operatorname{Aut}(G, S)$, a contradiction. A similar argument excludes the case where $G \cong \mathrm{M}_{11}$ or $\mathrm{M}_{23}$. Suppose that $G \cong \mathrm{A}_{p}$. Then $\operatorname{Inn}(G) \cong G \cong$ $\mathrm{A}_{p}$. Since $\operatorname{Inn}(G) \unlhd \operatorname{Aut}(G, S)$, then $\operatorname{Aut}(G, S) \cong \mathrm{A}_{p}$ or $\mathrm{S}_{p}$, and $\left|A_{1}\right|=2\left|\mathrm{~A}_{p}\right|$ or $2\left|\mathrm{~S}_{p}\right|$ respectively. Noting $p \geqslant 7$ is prime, by Lemma 7 , one can get that $A_{1} \cong \mathrm{S}_{p}$, and then $\operatorname{Aut}(G, S) \cong \mathrm{A}_{p}$ and $A=\left(G \cdot \mathrm{A}_{p}\right) \cdot \mathrm{C}_{2} \cong\left(\mathrm{A}_{p} \times \mathrm{A}_{p}\right) \cdot \mathrm{C}_{2}$. Then $\operatorname{Soc}(A)=G \times \mathrm{A}_{p} \cong \mathrm{A}_{p} \times \mathrm{A}_{p}$. Since $\operatorname{Soc}(A)$ is a characteristic subgroup of $A$ and $G \cong \mathrm{A}_{p}$ is a normal subgroup of $\operatorname{Soc}(A)$, then $G \unlhd A$. However, it contradicts to the assumption that $\Gamma$ is not a normal Cayley graph. Then $G \cong \operatorname{PSL}(d, q)$ for $p=\left(q^{d}-1\right) /(q-1)$, where $d \geqslant 2$. Along the same lines as the previous case, we can exclude this case.

Case (ii). Now assume that (a) of Lemma 5 holds, that is $A$ is an almost simple group with $G<L$ and $L$ is transitive on $V(\Gamma)$. Note that the valency of $\Gamma$ is prime $p$. Then, for $\alpha \in V(\Gamma), A_{\alpha}$ is primitive on $\Gamma(\alpha)$, so is $L_{\alpha}$ as $L_{\alpha} \unlhd A_{\alpha}$. It implies that $\Gamma$ is $L$-locally-primitive. Then $\Gamma=\Gamma\left(A, A_{\alpha}, g\right) \cong \Gamma\left(L, L_{\alpha}, t\right)$. Let $L=H D$ be a maximal factorization of $L$ for $G \leqslant H$ and $L_{\alpha} \leqslant D$, in particular, $L=G L_{\alpha}, G \cap L_{\alpha}=1$ and $|L|=|G|\left|L_{\alpha}\right|$. Now we assume that $L$ is not a classical simple group. Then the triples $\left(L, G, L_{\alpha}\right)$ are given in Table 7 of Lemma 9.

Since $|L|=|G|\left|L_{\alpha}\right|$, the calculation shows that only lines $1,4,7,9,10$ or 15 of Table 7 of Lemma 9 hold. In the following, we will analyze them one by one. Assume that $L \cong \mathrm{A}_{n}$ and $G \cong \mathrm{A}_{n-1}$, just as in line 1 of Table 7 . Then $\left|L_{\alpha}\right|=n$. It is shown in $[7$, Theorem 1.3] that there is a connected symmetric non-normal Cayley graph on $\mathrm{A}_{p-1}$ of valency $p$ for each prime $p \geqslant 7$. Then line 2 of Table 1 holds.

Now consider the line 4 of Table 7 . Since $|L|=|G|\left|L_{\alpha}\right|$, a straight forward calculation shows that $\left(L, G, L_{\alpha}\right) \cong\left(\mathrm{A}_{11}, \mathrm{~A}_{7}, \mathrm{M}_{11}\right)$ and $p=11$. With the help of MAGMA, no such 
graphs exist in this case. Assume that $\left(L, L_{\alpha}\right) \cong\left(\mathrm{A}_{p+1}, \mathrm{~A}_{p}\right)$ and $(p+1)|| G \mid$, as the line 6 of Table 7 . Then $|G|=p+1$, in particular, $\Gamma$ is the complete graph $\mathrm{K}_{p+1}$. Hence line 3 of Table 1 holds.

Assume that line 8 of Table 7 holds. Note that $|L|=|G|\left|L_{\alpha}\right|$. Then $\left(L, G, L_{\alpha}\right) \cong$ $\left(\mathrm{A}_{11}, \mathrm{M}_{11}, \mathrm{~A}_{7}\right)$ or $\left(\mathrm{A}_{12}, \mathrm{M}_{12}, \mathrm{~A}_{7}\right)$, in particular, $p=7$ and $\Gamma$ is 2-arc-transitive. With the help of MAGMA, neither $\Gamma\left(A_{11}, M_{11}, g\right)$ nor $\Gamma\left(A_{12}, M_{12}, g\right)$ exists. Suppose that line 10 of Table 7 holds. A straight forward calculation shows that $\left(L, G, L_{\alpha}\right) \cong\left(\mathrm{A}_{p+3}, \operatorname{PSL}(2, q), \mathrm{S}_{p}\right)$ for $q$ odd and $p=q-2$, and so the line 4 of Table 1 holds.

Suppose that the line 15 of Table 7 holds. Then $\left(L, G, L_{\alpha}\right) \cong\left(\mathrm{M}_{23}, \mathrm{M}_{22}, \mathrm{C}_{23}\right)$. Moreover, $p=23$ and $\Gamma$ is 1-regular. By Example 3, there does exist graph in this case. Hence line 1 of Table 1 holds, and thus Lemma 10 holds.

In the following Lemma, we will consider the case where Aut $\Gamma$ is not quasiprimitive on $V(\Gamma)$.

Lemma 11. Assume that $A$ is not quasiprimitive on $V(\Gamma)$. Then there exists an intransitive non-trivial normal subgroup $K$ of $A$ such that $A / K$ is almost simple with socle $\bar{L} \geqslant G K / K \cong G$. Moreover, for $\bar{\alpha} \in V\left(\Gamma_{K}\right)$, we have

(a) $\bar{L}$ is a classical simple group or $\left(\bar{L}, G, \bar{L}_{\bar{\alpha}}\right)$ lies in Table 2 ; or

(b) $\left(A, G, A_{\alpha}\right)$ lies in Table 3.

Proof. Since $A$ is not quasiprimitive, there exists a non-trivial maximal intransitive normal subgroup, say $K$. If $K$ has two orbits on $V(\Gamma)$, then $\Gamma$ is bipartite. Since $G$ is transitive on $V(\Gamma)$, then $G$ has a normal subgroup of index 2 , a contradiction. It follows that $K$ has at least $p+1$ orbits on $V(\Gamma)$. Let $\Gamma_{K}$ be the quotient graph of $\Gamma$ relative to $K$. Clearly, $\Gamma_{K}$ is arc-transitive with valency $p$. According to the maximum of $K$ and $\Gamma$ is locally primitive, we can conduct that the action of $A / K$ on $V\left(\Gamma_{K}\right)$ is quasiprimitive and $\Gamma_{K}$ is $A / K$-locally primitive, in particular, $(A / K)_{\bar{\alpha}}$ is given in Lemma 6 and 7 for $\bar{\alpha} \in V\left(\Gamma_{K}\right)$. Especially, Proposition 5 shows that there are only three cases in this situation:

(i). $A / K$ is almost simple, and $\operatorname{Soc}(A / K)$ contains $G K / K$ and is transitive on $V\left(\Gamma_{K}\right)$;

(ii). $A / K \cong \operatorname{AGL}(3,2), G \cong \operatorname{PSL}(2,7)$ and $\Gamma_{K} \cong \mathrm{K}_{8}$; or

(iii). $\operatorname{Soc}(A / K)=T \times T$, and $G K / K \cong G$ is a diagonal subgroup of $\operatorname{Soc}(A / K)$, where $T$ and $G$ are given in [9, Table 1$]$.

Case (i). Now suppose that $A / K$ is almost simple, just as (i). Write $\operatorname{Soc}(A / K)=\bar{L}$, which is a finite non-abelian simple group containing $\bar{G}=G K / K \cong G$. If $\bar{L}$ is regular on $V\left(\Gamma_{K}\right)$, then $\bar{L}=\bar{G}$ as $\bar{G} \leqslant \bar{L}$ is transitive on $\Gamma_{K}$. So $\bar{G}$ is regular on $V\left(\Gamma_{K}\right)$. It follows that $|V(\Gamma)|=|G|=|\bar{G}|=\left|V\left(\Gamma_{K}\right)\right|$, a contradiction. Hence $\bar{L}$ is not regular on $V\left(\Gamma_{K}\right)$. We claim that $\bar{L} \neq \bar{G}$. If not, then $\bar{L}=\bar{G}$, i.e., $G K / K$ is a characteristic subgroup of $A / K$, and so $G K$ is a characteristic subgroup of $A$. Noting that $G \unlhd G K$, we have $G \unlhd A$, and then $\Gamma$ is normal, a contradiction. Hence the claim holds. Then $\Gamma_{K}$ is $\bar{L}$-arctransitive, and so $\bar{L}$ is locally primitive as the valency of $\Gamma_{K}$ is prime, in particular, $\bar{L}_{\bar{\alpha}}$ is isomorphic to a group of Proposition 6 or 7 , where $\alpha \in V(\Gamma)$ and $\bar{\alpha} \in V\left(\Gamma_{K}\right)$. Further, $\bar{L}=\bar{L}_{\bar{\alpha}} \bar{G}$ with $\bar{G} \cap \bar{L}_{\bar{\alpha}}=\bar{G}_{\bar{\alpha}}$. Since $G$ is regular on $V(\Gamma)$ and $K$ is semiregular on $V(\Gamma)$, 
we have $K \cong \bar{G}_{\bar{\alpha}}$. Thus $|\bar{L}: \bar{G}|=\left|\bar{L}_{\bar{\alpha}}: \bar{G}_{\bar{\alpha}}\right|=\left|\bar{L}_{\bar{\alpha}}\right| /|K|$. We claim that $(A / K)_{\bar{\alpha}} \cong A_{\alpha}$. Note that $A_{\bar{\alpha}} / K=(A / K)_{\bar{\alpha}}$. By the Frattini argument, we have $A_{\bar{\alpha}}=K: A_{\alpha}$, i.e., $A_{\bar{\alpha}} / K \cong A_{\alpha}$. Hence $(A / K)_{\bar{\alpha}} \cong A_{\alpha}$, and so the claim holds. By Lemma $9,\left(\bar{L}, \bar{G}, \bar{L}_{\bar{\alpha}}\right)$ are given in Table 7. Since $|A|=\left|G A_{\alpha}\right|=|G|\left|A_{\alpha}\right|$ and $|K|=|A| /|A / K|$, we have $\left|A_{\alpha}\right| /|K|=|A / K| /|G|$. Note that $A_{\alpha} \cong(A / K)_{\bar{\alpha}}$ and $G \cong \bar{G}$. Then $\left|(A / K)_{\bar{\alpha}}\right| /|K|=|A / K| /|\bar{G}|$.

Suppose that $\bar{L}$ is not a classical simple group. Then by Lemma $9,\left(\bar{L}, \bar{G}, \bar{L}_{\bar{\alpha}}\right)$ are given in Table 7. In the following, we will analyze them one by one.

(1). Assume that $\bar{L} \cong \mathrm{A}_{n}$ and $\bar{G} \cong \mathrm{A}_{n-1}$, in particular, $n|| \bar{L}_{\bar{\alpha}} \mid$ and $p \mid n$ for $n \geqslant 6$, as line 1 of Table 7 . In [21, Theorem 1.1], it is shown that there exists a graph with $n=p=7$ and $\bar{L}_{\bar{\alpha}} \cong \mathrm{C}_{7}$. Hence line 1 of Table 2 holds. For a similar reason, lines 2-3 of Table 7 lead to that line 2-4 of Table 2 hold.

(2). Assume that $\left(\bar{L}, \bar{G}, \bar{L}_{\bar{\alpha}}\right) \cong\left(\mathrm{A}_{11}, \mathrm{~A}_{9}, \operatorname{PSL}(2,11)\right)$ or $\left(\mathrm{A}_{11}, \mathrm{~A}_{7}, \mathrm{M}_{11}\right)$ and $p=11$, just as line 4 of Table 7 . For $\left(\bar{L}, \bar{G}, \bar{L}_{\bar{\alpha}}\right) \cong\left(\mathrm{A}_{11}, \mathrm{~A}_{9}, \operatorname{PSL}(2,11)\right)$. Since $\operatorname{Soc}(A / K)=\bar{L} \cong \mathrm{A}_{11}$, we can conduct that $A / K \cong \mathrm{A}_{11}$ or $\mathrm{S}_{11}$, and so $(A / K)_{\bar{\alpha}} \cong \operatorname{PSL}(2,11)$ or $\operatorname{PSL}(2,11): \mathrm{C}_{2}$ respectively. On the other hand, $(A / K)_{\bar{\alpha}}$ is given in Proposition $7(\mathrm{~b})$, which gives that $(A / K)_{\bar{\alpha}} \cong \operatorname{PSL}(2,11)$, and so $A / K \cong \mathrm{A}_{11}$, i.e., $A / K=\bar{L}$. Furthermore, $\Gamma_{K}$ is $(\bar{L}, 2)$-arc transitive. By MAGMA, we have that the graph $\Gamma_{K}$ does not exist, a contradiction. Then along the same lines as the previous case we can exclude the cases when $\left(\bar{L}, \bar{G}, \bar{L}_{\bar{\alpha}}\right) \cong$ $\left(\mathrm{A}_{11}, \mathrm{~A}_{7}, \mathrm{M}_{11}\right)$ (which corresponding to line 4 of Table 7 ), and $\left(\mathrm{A}_{23}, \mathrm{~A}_{19}, \mathrm{M}_{23}\right)$ (which corresponding to line 5 of Table 7$)$.

(3). Assume that $\left(\bar{L}, \bar{L}_{\bar{\alpha}}\right) \cong\left(\mathrm{A}_{p+1}, \mathrm{~A}_{p}\right)$ and $(p+1)|| \bar{G} \mid$, just as line 6 of Table 7 . It is clear that $\Gamma_{K} \cong \mathrm{K}_{p+1}$. Hence line 5 of Table 2 holds. For a similar reason, line 7 and 10 of Table 7 gives line 6 and 7 of Table 2 respectively.

(4). Assume that $(\bar{L}, \bar{G}) \cong\left(\mathrm{A}_{11}, \mathrm{M}_{11}\right)$ or $\left(\mathrm{A}_{12}, \mathrm{M}_{12}\right)$, and $\bar{L}_{\bar{\alpha}} \cong \mathrm{A}_{7}$ or $\mathrm{S}_{7}$, in particular, $p=7$, just as line 8 of Table 7 . It is clear that $\mathrm{A}_{7}$ is 2-transitive on $\Gamma_{K}(\bar{\alpha})$. By Magma, we have that the graph $\Gamma_{K}$ does not exist, a contradiction. Hence this case does not occur.

(5). Assume that $(\bar{L}, \bar{G}) \cong\left(\mathrm{A}_{23}, \mathrm{M}_{23}\right)$ or $\left(\mathrm{A}_{24}, \mathrm{M}_{24}\right)$ and $\bar{L}_{\bar{\alpha}} \cong \mathrm{A}_{19}$ or $\mathrm{S}_{19}$, in particular, $p=19$, just as line 9 of Table 7 . Suppose that $(\bar{L}, \bar{G}) \cong\left(\mathrm{A}_{23}, \mathrm{M}_{23}\right)$. Note that $\left|\bar{L}_{\bar{\alpha}}\right| /|K|=$ $|\bar{L}| /|\bar{G}|=1267136462592000$ denoted by $m$, and $K \cong \bar{G}_{\bar{\alpha}} \leqslant(\bar{L})_{\bar{\alpha}}$, we have that $K$ is isomorphic to a subgroup of $\mathrm{A}_{19}$ or $\mathrm{S}_{19}$ with index $m$. Thus $|K|=48$ or 96 respects to $\bar{L}_{\bar{\alpha}} \cong \mathrm{A}_{19}$ or $\mathrm{S}_{19}$. Hence the first line of line 8 in Table 2 holds. For the same reason, the case where $(\bar{L}, \bar{G}) \cong\left(\mathrm{A}_{24}, \mathrm{M}_{24}\right)$ implies that line 8 of Table 2 holds.

(6). Assume that $(\bar{L}, \bar{G}) \cong\left(\mathrm{A}_{8}, \mathrm{~A}_{k}\right)$ for $k \in\{5,6,7\}$, and $\bar{L}_{\bar{\alpha}} \cong \operatorname{SL}(3,2)$ or $\operatorname{AGL}(3,2)$, in particular, $p=7$, as line 11 of Table 7 . By [13, Theorem 1.1], we have $\Gamma_{K}$ is $(\bar{L}, 2)$-arc transitive. With the help of MAGMA, there is no such graph $\Gamma_{K}$ exists, a contradiction.

(7). Assume that $\left(\bar{L}, \bar{G}, \bar{L}_{\bar{\alpha}}\right) \cong\left(\mathrm{M}_{12}, \mathrm{M}_{11}, \mathrm{M}_{11}\right)$ or $\left(\mathrm{M}_{12}, \mathrm{M}_{11}, \operatorname{PSL}(2,11)\right)$, and $p=11$ as line 12 of Table 7 . Suppose that $\left(\bar{L}, \bar{G}, \bar{L}_{\bar{\alpha}}\right) \cong\left(\mathrm{M}_{12}, \mathrm{M}_{11}, \mathrm{M}_{11}\right)$. Then $\Gamma_{K}$ is isomorphic to a complete graph $\mathrm{K}_{12}$. Note that $\operatorname{Soc}(A / K)=\bar{L} \cong \mathrm{M}_{12}$. Then $A / K \cong \mathrm{M}_{12}$ or $\mathrm{M}_{12}$. $\mathrm{C}_{2}$, and so $(A / K)_{\bar{\alpha}} \cong \mathrm{M}_{11}$ or $\mathrm{M}_{11} \cdot \mathrm{C}_{2}$. On the other hand, since $\Gamma_{K}$ is $A / K$-arc transitive graph of valency 11 , then $(A / K)_{\bar{\alpha}}$ is given in Proposition $7(\mathrm{~b})$, which shows that $A_{\alpha} \cong$ $(A / K)_{\bar{\alpha}} \cong \mathrm{M}_{11}$. It follows that $A / K=\bar{L} \cong \mathrm{M}_{12}$. Since $\left|\bar{L}_{\bar{\alpha}}\right| /|K|=|\bar{L}| /|\bar{G}|=12$ and $K \cong \bar{G}_{\bar{\alpha}} \leqslant(\bar{L})_{\bar{\alpha}} \cong \mathrm{M}_{11}$, we have that $K$ is isomorphic to a subgroup of $\mathrm{M}_{11}$ of index 12 . By [4, Page 18], $K \cong \operatorname{PSL}(2,11)$. On the other hand, the Schur multiplier $M\left(\mathrm{M}_{12}\right) \cong \mathrm{C}_{2}$ 
(see [4, Page 31] for example). Hence $A \cong K . A / K \cong \operatorname{PSL}(2,11) \cdot \mathrm{M}_{12} \cong \operatorname{PSL}(2,11) \times \mathrm{M}_{12}$. Hence $\left(A, G, A_{\alpha}\right) \cong\left(\operatorname{PSL}(2,11) \times \mathrm{M}_{12}, \mathrm{M}_{11}, \mathrm{M}_{11}\right)$, just as line 1 of Table 3 . Assume that $\left(\bar{L}, \bar{G}, \bar{L}_{\bar{\alpha}}\right) \cong\left(\mathrm{M}_{12}, \mathrm{M}_{11}, \operatorname{PSL}(2,11)\right)$. By Proposition $7(\mathrm{~b})$, we have $\Gamma_{K}$ is $(\bar{L}, 2)$-arc transitive. With the help of MAGMA, the graph $\Gamma_{K}$ does not exist, a contradiction.

(8). Suppose that $\left(\bar{L}, \bar{G}, \bar{L}_{\bar{\alpha}}\right) \cong\left(\mathrm{M}_{12}, \operatorname{PSL}(2,11), \mathrm{M}_{11}\right)$ and $p=11$ as line 13 of Table 7. Then $\Gamma_{K}$ is isomorphic to a complete graph $\mathrm{K}_{12}$. Note that $\bar{L}_{\bar{\alpha}} \cong \mathrm{M}_{11}$ and $\operatorname{Soc}(A / K)=$ $\bar{L} \cong \mathrm{M}_{12}$. Then $A / K \cong \mathrm{M}_{12}$ or $\mathrm{M}_{12} \cdot \mathrm{C}_{2}$, and so $(A / K)_{\bar{\alpha}} \cong \mathrm{M}_{11}$ or $\mathrm{M}_{11} \cdot \mathrm{C}_{2}$ respectively. On the other hand, since $\Gamma_{K}$ is $A / K$-arc transitive graph of valency 11 , then $(A / K)_{\bar{\alpha}}$ is given in Proposition $7(\mathrm{~b})$, which shows that $(A / K)_{\bar{\alpha}} \cong \mathrm{M}_{11}$. It follows that $A_{\alpha} \cong(A / K)_{\bar{\alpha}} \cong$ $\mathrm{M}_{11}$ and $A / K \cong \bar{L} \cong \mathrm{M}_{12}$. Noting $G \cong \bar{G} \cong \operatorname{PSL}(2,11)$, we have $\left|(A / K)_{\bar{\alpha}}\right| /|K|=$ $|A / K| /|\bar{G}|=144$. Since $K \cong \bar{G}_{\bar{\alpha}} \leqslant(A / K)_{\bar{\alpha}} \cong \mathrm{M}_{11}$, we have $K$ is isomorphic to a subgroup of $\mathrm{M}_{11}$ of index 144. By [4, Page 18], $K \cong \mathrm{C}_{11}: \mathrm{C}_{5}$ and the Schur multiplier $M\left(\mathrm{M}_{12}\right) \cong \mathrm{C}_{2}$, and hence $A \cong K \cdot A / K \cong\left(\mathrm{C}_{11}: \mathrm{C}_{5}\right) \cdot \mathrm{M}_{12}$. With the help of GAP, we have $\operatorname{Aut}\left(\mathrm{C}_{11}: \mathrm{C}_{5}\right) \cong\left(\mathrm{C}_{11}: \mathrm{C}_{5}\right): \mathrm{C}_{2}$. Thus $\left(\mathrm{C}_{11}: \mathrm{C}_{5}\right) \cdot \mathrm{M}_{12} \cong\left(\mathrm{C}_{11}: \mathrm{C}_{5}\right) \times \mathrm{M}_{12}$. Thereby, $\left(A, G, A_{\alpha}\right) \cong$ $\left(\left(\mathrm{C}_{11}: \mathrm{C}_{5}\right) \times \mathrm{M}_{12}, \operatorname{PSL}(2,11), \mathrm{M}_{11}\right)$, just as line 2 of Table 3 .

(9). Assume that $\left(\bar{L}, \bar{G}, \bar{L}_{\bar{\alpha}}\right) \cong\left(\mathrm{M}_{12}, \mathrm{~A}_{5}, \mathrm{M}_{11}\right)$ as line 14 of Table 7 , in particular, $p=11$ and $\Gamma_{K} \cong \mathrm{K}_{12}$. Note that $\bar{L}_{\bar{\alpha}} \cong \mathrm{M}_{11}$ and $\operatorname{Soc}(A / K)=\bar{L} \cong \mathrm{M}_{12}$. Then $A / K \cong \mathrm{M}_{12}$ or $\mathrm{M}_{12} \cdot \mathrm{C}_{2}$, and so $(A / K)_{\bar{\alpha}} \cong \mathrm{M}_{11}$ or $\mathrm{M}_{11} \cdot \mathrm{C}_{2}$. On the other hand, since $\Gamma_{K}$ is $A / K$-arc transitive graph of valency 11 , then $(A / K)_{\bar{\alpha}}$ is given in Proposition $7(\mathrm{~b})$, which shows that $(A / K)_{\bar{\alpha}} \cong \mathrm{M}_{11}$. It follows that $A_{\alpha} \cong(A / K)_{\bar{\alpha}} \cong \mathrm{M}_{11}$ and $A / K=\bar{L} \cong \mathrm{M}_{12}$. Note that $G \cong \bar{G} \cong \mathrm{M}_{11}$, we have $\left|(A / K)_{\bar{\alpha}}\right| /|K|=|A / K| /|G|=\left|\mathrm{M}_{12}\right| /\left|\mathrm{A}_{5}\right|=2^{4} \cdot 3^{2} \cdot 11$. Since $K \cong \bar{G}_{\bar{\alpha}} \leqslant(A / K)_{\bar{\alpha}} \cong \mathrm{M}_{11}$, we have $K$ is isomorphic to a subgroup of $\mathrm{M}_{11}$ of index $2^{4} \cdot 3^{2} \cdot 11$. By [4, Page 18], $K \cong \mathrm{C}_{5}$ and $M\left(\mathrm{M}_{12}\right) \cong \mathrm{C}_{2}$, and hence $A \cong K . A / K \cong$ $\mathrm{C}_{5} \cdot \mathrm{M}_{12} \cong \mathrm{C}_{5} \times \mathrm{M}_{12}$. Hence $\left(A, G, A_{\alpha}\right) \cong\left(\mathrm{C}_{5} \times \mathrm{M}_{12}, \mathrm{~A}_{5}, \mathrm{M}_{11}\right)$, just as line 3 of Table 3 .

(10). Assume that $(\bar{L}, \bar{G}) \cong\left(\mathrm{M}_{23}, \mathrm{M}_{22}\right)$ and $\bar{L}_{\bar{\alpha}} \cong \mathrm{C}_{23}: \mathrm{C}_{11}$ or $\mathrm{C}_{23}$, as line 15 of Table 7, in particular, $p=23$. Note that $\operatorname{Soc}(A / K)=\bar{L} \cong \mathrm{M}_{23}$ and $\operatorname{Out}\left(\mathrm{M}_{23}\right)=$ 1. Then $A / K \cong \mathrm{M}_{23}$, and so $A_{\alpha} \cong(A / K)_{\bar{\alpha}}=\bar{L}_{\bar{\alpha}} \cong \mathrm{C}_{23}: \mathrm{C}_{11}$ or $\mathrm{C}_{23}$. In particular, $\left|(A / K)_{\bar{\alpha}}\right| /|K|=|A / K| /|\bar{G}|=23$. Since $K \cong \bar{G}_{\bar{\alpha}} \leqslant(A / K)_{\bar{\alpha}}$ of index 23 and $K \neq 1$, then $\bar{L}_{\bar{\alpha}} \cong \mathrm{C}_{23}: \mathrm{C}_{11}$ and $K \cong \mathrm{C}_{11}$. By [4, Page 71 ], the Schur multiplier $M\left(\mathrm{M}_{23}\right)=1$, and so $A \cong \mathrm{C}_{11} \cdot \mathrm{M}_{23} \cong \mathrm{C}_{11} \times \mathrm{M}_{23}$. Hence line 4 of Table 3 holds.

(11). Assume that $(\bar{L}, \bar{G}) \cong\left(\mathrm{M}_{24}, \mathrm{M}_{23}\right)$ and $\bar{L}_{\bar{\alpha}} \cong \mathrm{SL}(3,2)$ or $\mathrm{C}_{2}^{6}:\left(\mathrm{SL}(3,2) \times \mathrm{S}_{3}\right)$, as line 16 of Table 7 , in particular, $p=7$. Suppose that $\bar{L}_{\bar{\alpha}} \cong \mathrm{C}_{2}^{6} \times\left(\operatorname{SL}(3,2) \times \mathrm{S}_{3}\right)$. By Lemma 6 , we have $\Gamma_{K}$ is $(\bar{L}, 2)$-arc transitive. However, by MAGMA, we have that the graph $\Gamma_{K}$ does not exist, a contradiction. If $\bar{L}_{\bar{\alpha}} \cong \mathrm{SL}(3,2)$, then $P:=\bar{L}_{\bar{\alpha} \bar{\beta}} \cong \mathrm{S}_{4}$ for $\bar{\beta} \in \Gamma_{K}(\bar{\alpha})$. With the help of GAP, we have $N:=\mathrm{N}_{\bar{L}}(P) \cong \mathrm{S}_{3} \times \mathrm{S}_{4}$ and $\left\langle\bar{L}_{\bar{\alpha}}, N\right\rangle<\mathrm{M}_{24}$. It contradicts to the assumption that $\Gamma_{K}$ is connected.

(12). Assume that $\left(\bar{L}, \bar{G}, \bar{L}_{\bar{\alpha}}\right) \cong\left(\mathrm{M}_{24}, \operatorname{PSL}(2,23), \mathrm{M}_{23}\right)$ as line 17 of Table 7 , in particular, $p=23$ and $\Gamma_{K} \cong \mathrm{K}_{24}$. Note that $\bar{L}_{\bar{\alpha}} \cong \mathrm{M}_{23}$ and $\operatorname{Soc}(A / K)=\bar{L} \cong \mathrm{M}_{24}$. Then $A / K \cong \mathrm{M}_{24}$ and so $A_{\alpha} \cong(A / K)_{\bar{\alpha}} \cong \mathrm{M}_{23}$. Note that $G \cong \bar{G} \cong \operatorname{PSL}(2,23)$, we have $\left|(A / K)_{\bar{\alpha}}\right| /|K|=|A / K| /|G|=\left|\mathrm{M}_{24}\right| /|\operatorname{PSL}(2,23)|=2^{7} \cdot 3^{2} \cdot 5 \cdot 7$. Since $K \cong \bar{G}_{\bar{\alpha}} \leqslant$ $(A / K)_{\bar{\alpha}} \cong \mathrm{M}_{23}$, we have $K$ is isomorphic to a subgroup of $\mathrm{M}_{23}$ of index $2^{7} \cdot 3^{2} \cdot 5 \cdot 7$. By [4, Page 71$], K \cong \mathrm{C}_{23}: \mathrm{C}_{11}$ and $M\left(\mathrm{M}_{24}\right)=1$, and hence $A \cong K \cdot A / K \cong\left(\mathrm{C}_{23}: \mathrm{C}_{11}\right) \cdot \mathrm{M}_{24} \cong$ $\left(\mathrm{C}_{23}: \mathrm{C}_{11}\right) \times \mathrm{M}_{24}$. Hence $\left(A, G, A_{\alpha}\right) \cong\left(\left(\mathrm{C}_{23}: \mathrm{C}_{11}\right) \times \mathrm{M}_{24}, \mathrm{PSL}(2,23), \mathrm{M}_{23}\right)$, just as line 5 of 
Table 3.

Case (ii). Assume that (ii) occurs, i.e., $A / K \cong \operatorname{AGL}(3,2), G \cong \operatorname{PSL}(2,7)$ and $\Gamma_{K} \cong \mathrm{K}_{8}$. Since $\bar{G} \cong G$ is transitive on $V\left(\Gamma_{K}\right)$, then $|G| / 8=\left|\bar{G}_{\bar{\alpha}}\right|$, in particular, the index of $\bar{G}_{\bar{\alpha}}$ in $G$ is 8 . It follows that $\bar{G}_{\bar{\alpha}} \cong \mathrm{C}_{7}: \mathrm{C}_{3}$. Since $G$ is regular and $K$ is semiregular on $V(\Gamma)$, then $\bar{G}_{\bar{\alpha}} \cong K$. Hence $A \cong K \cdot A / K \cong\left(\mathrm{C}_{7}: \mathrm{C}_{3}\right) \cdot \operatorname{AGL}(3,2)$. Note that $\operatorname{AGL}(3,2) \cong$ $\mathrm{C}_{2}^{3}$ :SL(3,2) with $\mathrm{C}_{2}^{3}$ being the unique minimal normal subgroup. By [4], $M(\mathrm{SL}(3,2)) \cong \mathrm{C}_{2}$. Note that $\operatorname{Aut}\left(\mathrm{C}_{7}: \mathrm{C}_{3}\right) \cong\left(\mathrm{C}_{7}: \mathrm{C}_{3}\right): \mathrm{C}_{2}$, we have $A \cong\left(\mathrm{C}_{7}: \mathrm{C}_{3}\right) \times \operatorname{AGL}(3,2)$. On the other hand, since $|A / K|=8\left|(A / K)_{\bar{\alpha}}\right|$ and $A / K=\operatorname{AGL}(3,2) \cong \mathrm{C}_{2}^{3}: \operatorname{SL}(3,2)$, then $(A / K)_{\bar{\alpha}} \cong$ $\mathrm{SL}(3,2)$. It follows that $A_{\alpha} \cong(A / K)_{\bar{\alpha}} \cong \mathrm{SL}(3,2)$. Then Lemma 11 holds in this case.

Case (iii). Assume that (iii) occurs, i.e., $\operatorname{Soc}(A / K)=T \times T$, and $G K / K \cong G$ is a diagonal subgroup of $\operatorname{Soc}(A / K)$, where $T$ and $G$ are given in [9, Table 1]. Then $\Gamma_{K}$ is $\bar{L}$-arc transitive and $\bar{L}_{\bar{\alpha}}$ is primitive on $\Gamma_{K}(\bar{\alpha})$. So $\bar{L}_{\bar{\alpha}}$ is given in Lemma 6 and 7 , in particular, $\left(|\operatorname{Soc}(A / K)| /\left|V\left(\Gamma_{K}\right)\right|\right)_{p}=\left|\bar{L}_{\alpha}\right|_{p}=p$ for $p \geqslant 7$. However a calculation on the index of $\left|V\left(\Gamma_{K}\right)\right|$ in $\operatorname{Soc}(A / K)$ shows that $\left(|\operatorname{Soc}(A / K)| /\left|V\left(\Gamma_{K}\right)\right|\right)_{p} \geqslant p^{2}$, a contradiction.

This finishes the proof of Lemma 11.

The proof of Theorem 1: The Theorem 1 follows immediately from Lemma 10 (which gives (a) of Theorem 10) and Lemma 11 (which gives (b) of Theorem 10).

\section{Acknowledgements}

The authors acknowledge the use of the MAGMA and GAP computational package, which helped show some of the results given in this paper.

\section{References}

[1] B. Baumeister, Primitive permutation groups with a regular subgroup, J. Algebra, 310(2): 569-618, 2007.

[2] J. N. Bray, D. F. Holt and C. M. Roney-Dougal, The Maximal Subgroups of the Low-Dimensional Finite Classical Groups, Cambridge University Press, New York, 2013.

[3] P. J. Cameron, Finite permutation groups, Cambridge University Press, 1999.

[4] J. H. Conway, R. T. Curtis, S. P. Norton, R. A. Parker and R. A. Wilson, Atlas of Finite Groups, Clarendon Press, Oxford, 1985. (http://brauer .maths .qmul . ac . uk/Atlas/v3/).

[5] J.-L. Du, Y.-Q. Feng and J.-X. Zhou, Pentavalent symmetric graphs admitting vertex-transitive non-abelian simple groups, European J. Combin., 63: 134-145, 2017.

[6] X. G. Fang, C. H. Li, J. Wang and M. Y. Xu, On cubic Cayley graphs of finite simple groups, Discrete Math., 244(1-3): 67-75, 2002.

[7] X. G. Fang, X. Ma and J. Wang, On locally primitive Cayley graphs of finite simple groups, J. Combin. Theory Ser. A, 118: 1039-1051, 2011. 
[8] X. G. Fang and C. E. Praeger, Finite two-arc transitive graphs admitting a Suzuki simple group, Comm. Algebra, 27(8): 3727-3754, 1999.

[9] X. G. Fang, C. E. Praeger and J. Wang, On the automorphism group of Cayley graphs of finite simple groups, J. London Math. Soc. (2), 66(3): 563-578, 2002.

[10] C. D. Godsil, On the full automorphism group of a graph, Combinarorica, 1: 243-256, 1981.

[11] M. Giudici, Factorisations of sporadic simple groups, J. Algebra, 304(1): 311-323, 2006.

[12] S.-T. Guo, H. Hou and Y. Xu, A note on solvable vertex stabilizers of $s$-transitive graphs of prime valency, Czechoslovak Math. J., 65: 781-785, 2015.

[13] S.-T. Guo, Y. Li and X.-H. Hua, (G,s)-transitive graphs of valency 7, Algebra Colloq., 23(3): 493-500, 2016.

[14] C. Hering, M. W. Liebeck, and J. Saxl, The factorizations of the finite exceptional groups of lie type, J. Algebra, 106(2): 517-527, 1987.

[15] W. M. Kantor, k-homogeneous groups, Math. Z., 124: 261-265, 1972.

[16] P. Kleidman and M. Liebeck, The subgroup structure of the finite classical groups, Cambridge University Press, New York, 1990.

[17] C. H. Li, Isomorphims of finite Cayley graphs, PhD Thesis, The University of Western Australia, 1996.

[18] C. H. Li, The finite primitive permutation groups containing an abelian regular subgroup, Proc. London Math. Soc., 87(3): 725-747, 2003.

[19] C. H. Li, Á. Seress and S. J. Song, s-arc-transitive graphs and normal subgroups, J. Algebra, 421: 331-348, 2015.

[20] J. J. Li, B. Ling and G. D. Liu, A characterisation on arc-transitive graphs of prime valency, Appl. Math. Comput., 325: 227-233, 2018.

[21] J. J. Li, G. R. Zhang and B. Ling, 1-regular Cayley graphs of valency 7, Bull. Aust. Math. Soc., 88(3): 479-485, 2013.

[22] M. W. Liebeck, C. E. Praeger and J. Saxl, The maximal factorizations of the finite simple groups and their automorphism groups, Mem. Amer. Math. Soc., 86, no. 432, 1990.

[23] M. W. Liebeck, C. E. Praeger and J. Saxl, Transitive subgroups of primitive permutation groups, J. Algebra 234(2): 291-361, 2000.

[24] M. Y. Xu, Automorphism groups and isomorphisms of Cayley graphs, Discrete Math. 182: 309-319, 1998.

[25] S. J. Xu, X. G. Fang, J. Wang and M. Y. Xu, On cubic s-arc transitive Cayley graphs of finite simple groups, European J. Combin. 26(1): 133-143, 2005.

[26] C. Zhang and X. G. Fang, A note on the automorphism groups of cubic Cayley graphs of finite simple groups, Discrete Math., 310(21): 3030-3032, 2010. 